\title{
Funciones vectoriales y curvas en el espacio mediante software de geometría dinámica: Una experiencia sobre la génesis instrumental de profesores en formación
}

\author{
Cesar Martínez Hernández ${ }^{1}$ \\ cmartinez7@ucol.mx \\ https://orcid.org/0000-0002-9958-8152 \\ Rodolfo Rangel Alcántar ${ }^{1}$ \\ rodolfo1@ucol.mx \\ https://orcid.org/0000-0002-4130-054X \\ José Marcos López Mojica ${ }^{2}$ \\ mojicajm@gmail.com \\ https://orcid.org/0000-0002-7330-9979 \\ Norma Angélica Barón Ramírez ${ }^{1}$ \\ norma@ucol.mx \\ https://orcid.org/0000-0002-7555-5073 \\ ${ }^{1}$ Universidad de Colima (U. de C, México) \\ ${ }^{2}$ Universidad Autónoma de Guerrero (UAGro, México)
}

Recibido: 31/05/2020 Aceptado: 08/07/2020

\begin{abstract}
Resumen
En este reporte se presenta la actividad matemática en que se enrolan profesores en formación en torno al trabajo con funciones vectoriales, particularmente respecto a la visualización de curvas en el espacio mediante GeoGebra. El estudio de naturaleza cualitativa y de tipo exploratorio, se sustentó en los elementos teóricos de la aproximación instrumental del uso de herramientas tecnológicas, en su enfoque ergonómico. Los resultados revelan cómo los profesores utilizan herramientas particulares del software de Geometría Dinámica GeoGebra como instrumento que les permite la reflexión matemática sobre curvas y vectores en el espacio. El estudio se enfoca en el análisis de la génesis instrumental a nivel personal respecto al uso del arrastre a través de deslizadores. Las formas de uso de la herramienta tecnológica por parte de los futuros profesores para visualizar la curva que genera la función vectorial se pueden clasificar en tres: el uso de deslizadores para generar una familia de vectores y el rastro para visualizar la curva generada; el uso del registro numérico mediante la hoja de cálculo y la conversión hacia la representación gráfica; el uso del deslizador y el rastro para visualizar tanto la curva generada como los vectores que la producen.

Palabras clave: Funciones vectoriales; Curvas en el espacio; GeoGebra; Génesis instrumental personal.
\end{abstract}

Vector functions and space curves in dinamyc geometry software: An experience on the intrumental genesis of preservice mathematics teachers 


\begin{abstract}
In this report we show the kind of mathematical activity in which preservice mathematics teachers are enrolled when they face with vector functions, especially regarding the visualization of space curves. The qualitative in nature and exploratory research, was based on the theoretical elements of the instrumental approach to tool use, on its ergonomic direction. The results we present disclose how preservice teachers use particular GeoGebra tools as instruments. This kind of use of digital tools, allows preserve teachers to engage in a rich mathematical activity on vector functions and its space curves. Our study focuses on an analysis of the instrumental genesis at a personal level, regarding the use of dragging tool in an implicit way through the use of sliders. Three different types of use of specific tools of GeoGebra were identified in their mathematical activity related to visualize the space curve of a vector function: the use of a slider to visualize a family of points generated by each value of the slider associated to each vector function and to generate de space curve using the trace tool; the use of the spreadsheet in order to work in the numerical register and to represent a family of vectors and its geometric representation; finally, the use of the slider and the trace tool to visualize both the points associated to a family of vectors and the vectors in itself.
\end{abstract}

Keywords: Vector functions; Space curves; GeoGebra; Personal instrumental genesis.

\title{
Funções vetoriais e curvas no espaço usando software de geometria dinâmica: uma experiência sobre a gênese instrumental de professores em Formação
}

\section{Resumo}

Este artigo apresenta a atividade matemática de professores em formação quando tratam de funções vetoriais, principalmente no que diz respeito à visualização de curvas no espaço utilizando o GeoGebra. O estudo de natureza qualitativa e exploratória baseou-se nos elementos teóricos da abordagem instrumental à utilização de ferramentas tecnológicas, na sua abordagem ergonómica. Os resultados revelam como os professores utilizam determinadas ferramentas do software GeoGebra como um instrumento que lhes permite refletir matematicamente sobre curvas e vetores no espaço. $\mathrm{O}$ estudo enfatiza a análise da gênese instrumental em um nível pessoal quanto ao uso de arrastar por meio de controles deslizantes. As formas de utilização da ferramenta tecnológica pelos futuros professores para visualizar a curva gerada pela função vetorial podem ser classificadas em três: o uso de controles deslizantes para gerar uma família de vetores e o traço para visualizar a curva gerada; o uso do registro numérico por meio da folha de cálculo e a conversão para a representação gráfica e o uso do controle deslizante e do traço para visualizar a curva gerada e os vetores que a produzem.

Palavras-chave: Funções Vetoriais; Curvas Espaciais; GeoGebra; Gênese instrumental pessoal.

\section{Antecedentes y problematización}

El uso de tecnología en la educación matemática es un tópico multifacético, uno de tales intereses versa sobre la formación de profesores (Hegedus, Laborde, Brady, Dalton, Siller, Tabach, Trgalova \& Moreno-Armella, 2017). A este respecto Huang y Zbiek (2017), basados 
en una revisión de la literatura agrupan las investigaciones sobre el uso de tecnología y la formación de profesores de la siguiente manera: estudios sobre la tecnología y contenidos matemáticos; sobre tecnología y aspectos pedagógicos, así como estudios sobre la práctica del futuro profesor en medios tecnológicos.

Respecto a contenidos matemáticos y la tecnología, de acuerdo con Huang y Zbiek (2017), las investigaciones de este grupo pueden a su vez categorizarse en los siguientes tipos: el uso de tecnología para promover en los futuros profesores la comprensión de contenidos matemáticos, para incrementar su desempeño en el aprendizaje matemático o bien para promover nuevas actitudes respecto a la enseñanza y aprendizaje mediante tecnología.

La categorización propuesta por Huang y Zbieck (2017) concuerda en cierto sentido con modelos sobre los tipos de conocimiento que debe poseer y desarrollar el profesor de matemáticas, por ejemplo el Conocimiento Tecnológico Pedagógico del Contenido (TPACK, por sus siglas en inglés) (Mishra \& Koehler, 2006), el cual refiere a la intersección entre conocimientos sobre la tecnología, el contenido y la pedagogía; a la vez, concuerda con el planteamiento de Lobo da Costa, Galvao y Prado (2017) respecto a que un conocimiento como el TPACK implica que el profesor construya o reconstruya su conocimiento matemático en medios tecnológicos. En este mismo sentido, Martínez-Hernández \& Ulloa-Azpeitia (2017) indican que los profesores y futuros profesores deben tener oportunidad de reflexionar y desarrollar su propio conocimiento matemático, y no sólo indicarles el posible potencial de su uso para la enseñanza y aprendizaje de las matemáticas, el propio profesor en formación es quien debe tener oportunidad de aprender en medios tecnológicos.

Los planteamientos de Lobo da Costa et al. (2017) así como de Martínez-Hernández y Ulloa-Azpeitia (2017), están en línea con la categorización referida al uso de tecnología para promover la comprensión matemática de futuros profesores. De acuerdo con Huang y Zbieck (2017), en la literatura especializada sobresalen aquellos estudios que refieren al uso de ambientes tecnológicos dinámicos o interactivos para promover la comprensión matemática de profesores en formación. Por ejemplo, Cory y Garofolo (2011) reportan sobre el cambio en la concepción de límites de secuencias de profesores en formación a partir de la representación visual de su definición formal. Por su parte, Lobo da Costa et al. (2017) reportan sobre la reconstrucción de conocimientos trigonométricos de profesores través de software de geometría 
dinámica. En este mismo tipo de ambiente tecnológico, Martínez-Hernández y Ulloa-Azpeitia (2017), indican que el uso de tecnología, con el diseño de tareas adecuadas, permite a profesores desarrollar aspectos técnicos y teóricos de la matemática en torno al teorema del valor medio.

Modelos como el TPACK (Mishra \& Koehler, 2006), desde nuestro punto de vista, dejan fuera aspectos necesarios a considerar sobre la enseñanza de las matemáticas. Por ejemplo, lo planteado por Lobo da Costa et al. (2017) en cuanto a considerar que el desarrollo de un conocimiento de tipo TPACK por parte del profesor o futuro profesor, implica que él mismo se apropie de los recursos tecnológicos; ello será posible si construye o reconstruye su conocimiento matemático en estos ambientes tecnológicos, es decir, a partir de proveer de experiencias sobre la actividad matemática al profesor en servicio o en formación (MartínezHernández \& Ulloa-Azpeitia, 2017), o como lo mencionan Clark-Wilson, Guilles, Cusi, Goos, Haspekian, Robutti y Thomas (2014), el profesor requiere apropiarse de la tecnología antes de promoverla con sus alumnos. Esta característica de la actividad matemática mediada por recursos tecnológicos implica la instrumentación del artefacto tecnológico (Lobo da Costa et al., 2017).

Los estudios referidos a la instrumentación de artefactos tecnológicos por parte del profesor, pretenden explicar el proceso de apropiación de herramientas tecnológicas, es decir, la llamada Génesis Instrumental (Drijvers \& Trouche, 2008, Trouche, 2005). Sin embargo, referida al profesor, Haspekian (2011) postula lo que se conoce como Doble Génesis Instrumental, en la cual, por un lado, se distingue una génesis instrumental a nivel personal (en su propio aprendizaje matemático); por otro, se distingue la génesis instrumental a nivel profesional (referida a la apropiación de la herramienta tecnológica para la enseñanza). La categorización propuesta por Huang y Zbiek (2017), sobre el uso de la tecnología para promover la comprensión matemática de profesores en formación, está en línea con la génesis instrumental a nivel personal.

Los estudios sobre la génesis instrumental de profesores, por un lado, destacan la importancia del uso de herramientas tecnológicas. Por ejemplo, Bozkurt, Uygan, y Turgut (2018), reportan sobre las acciones instrumentadas que desarrolla un profesor en formación sobre una construcción geométrica en un ambiente de geometría dinámica, y, por otro, respecto al desarrollo profesional de profesores. Investigaciones recientes han indicado la complejidad 
de alcanzar el desarrollo de la génesis instrumental, a este respecto, Santos y Acuña (2015) señalan que profesores de bachillerato desarrollan parcialmente la génesis instrumental sobre el uso de tabletas para la enseñanza. De acuerdo con estos investigadores, los profesores sólo desarrollan el llamado proceso de instrumentalización, no así el de instrumentación. Sin embargo, el proceso de instrumentación al que refieren Santos y Acuña (2015) es un proceso de instrumentación sobre la enseñanza, no para el propio aprendizaje matemático de los profesores.

Posiblemente, una de las dificultades para no lograr plenamente la Génesis instrumental (a nivel profesional) se deba a la poca experiencia de los profesores con la génesis a nivel personal. No debería ser este el caso con profesores en formación, ya que es un escenario adecuado para desarrollar en ellos conocimientos matemáticos mediados por herramientas tecnológicas. Así, en este trabajo proponemos como necesario e indispensable distinguir claramente la génesis instrumental personal de la profesional. Dado el escenario de la formación inicial del profesor de matemáticas, ella o él puede tener oportunidades de apropiación de la herramienta tecnológica tanto para su aprendizaje matemático, como para la reflexión sobre el potencial de tales herramientas en su futura práctica en el aula de matemáticas (ya sea en escenarios reales o hipotéticos). Así, en este trabajo damos cuenta de una experiencia sobre el uso de GeoGebra por parte de futuros profesores y su reflexión matemática en el tema de funciones vectoriales y curvas en el espacio. La pregunta que guio la investigación es: ¿qué procesos de la génesis instrumental manifiestan profesores en formación al hacer uso de GeoGebra en el tema de funciones vectoriales y curvas en el espacio?

\section{Referente teórico}

\section{Conocimiento Matemático Digital para la Enseñanza y la Génesis Instrumental}

En un análisis de diferentes modelos teóricos sobre el tipo de conocimientos necesarios para la enseñanza de las matemáticas con tecnología, Hegedus, et al. (2017) reconocen que dos de los referentes que sobresalen son el modelo TPACK (Mishra \& Koehler, 2006), así como la Aproximación Instrumental (Drijvers \& Trouche, 2008; Rabardel, 2002; Trouche, 2005). Por su parte, Tabach y Trgalová (2018) proponen un modelo de Competencias Digitales para la Enseñanza de las Matemáticas con Tecnología, el cual, por un lado, adapta el modelo TPACK, para hacerlo específico al dominio matemático, el cual nombran como Conocimimiento 
Matemático Digital para la Enseñanza (MDKT, por sus siglas en inglés) y por otro, reconoce la importancia de la doble génesis instrumental (Haspeskian, 2011). Dado que el presente reporte se enfoca en la reflexión matemática de profesores en formación mediada por un software de Gometría Dinámica, la Génesis Instrumental a nivel personal es el constructo teórico suficiente para sustentar el diseño y análisis de este trabajo.

Con base en la ergonomía cognitiva de Rabardel (2002), la Aproximación Instrumental del uso de herramientas tecnológicas (Drijvers \& Trouche, 2008; Trouche, 2005) es un doble proceso dialéctico que explica la transformación de un artefacto (tecnológico) en un instrumento para la actividad matemática que el usuario desarrolla a partir de la herramienta digital. Trouche (2005) define al artefacto como una herramienta con la cual un sujeto puede llevar a cabo cierta actividad, esta puede carecer de sentido para el usuario cuando no identifica potencialidades o formas de resolver un problema con dicho artefacto, sin embargo, una vez que el usuario es consciente sobre la extensión de sus capacidades cognitivas mediadas por el artefacto, este se convierte en instrumento, ello implica el desarrollo de formas de uso del artefacto, es decir, de esquemas (Drijvers \& Trouche, 2008). Así, un instrumento emerge en la actividad matemática, cuando existe una relación con sentido entre artefacto y el usuario. Por ello, se reconoce que un instrumento consiste del artefacto y de esquemas mentales que el sujeto desarrolla al hacer uso del artefacto dentro de la actividad matemática. Un artefacto se convierte en un instrumento mediante un proceso llamado Génesis Instrumental, el cual consiste en dos procesos bidireccionales: instrumentalización e instrumentación (Drijvers \& Trouche, 2008, Trouche, 2005).

La instrumentalización es un proceso dirigido del individuo hacia el artefacto, ya que las preferencias y concepciones del usuario influyen en las formas en que el sujeto usa el artefacto (Drijvers \& Trouche, 2008). Por ello, los conocimientos matemáticos que posea el individuo le guiarán en la forma de usar el artefacto (Kieran \& Drijvers, 2006). Por otro lado, la instrumentación es un proceso dirigido del artefacto hacia el sujeto, este proceso refiere a las posibilidades y restricciones que el artefacto ofrece al usuario, por lo que a partir de éstas, influye en las formas de pensar del usuario, es decir, desarrolla esquemas (de uso y de acción instrumentada), en este trabajo interesan las segundas. 
De esta manera, las formas de uso del artefacto (i.e., esquemas desarrollados), o como lo indican Bozkurt, Uygan, y Turgut (2018), las estrategias asociadas al uso de herramientas específicas del software de geometría dinámica (i.e., acciones instrumentadas) son el foco de atención de la actividad matemática del usuario del artefacto.

\section{Método}

\section{Participantes y toma de datos}

Dado que en este trabajo se reporta en torno al desarrollo de las formas de uso del artefacto por parte de futuros profesores, la presente investigación es de naturaleza cualitativa y de tipo exploratorio (Vasilachis, 2006). El estudio se llevó a cabo con 16 profesores en formación de una Universidad Pública de México. Al momento de la toma de datos, los participantes cursaban el octavo semestre de su formación universitaria. La recopilación de información se llevó a cabo durante su curso regular de Cálculo en varias variables, específicamente en el tema de funciones vectoriales y curvas en el espacio. Los 16 participantes, tenían experiencia en el manejo del Software de Geometría Dinámica (GeoGebra), de manera sistemática, durante al menos un año en cursos previos de cálculo. Sin embargo, fue la primera ocasión en que abordaron el uso de la ventana 3D para visualizar vectores en el espacio tridimensional.

Para la toma de datos, se suministró a cada participante la Tarea diseñada para tal fin, la cual respondieron de forma individual en horario fuera de clase. Una vez resuelta la Tarea, se llevó a cabo una entrevista semiestructurada para discutir y socializar las respuestas de cada participante. La entrevista semiestructurada se llevó a cabo en línea, conducida por el primer autor de este trabajo, a través de videoconferencia, ya que la toma de datos se desarrolló durante el periodo de confinamiento por la contingencia sanitaria debido a la pandemia mundial asociada al COVID-19. De esta manera, los participantes tuvieron oportunidad de resolver de manera individual la tarea propuesta, antes de la discusión grupal en línea. En cuanto al uso sistemático de GeoGebra, los 16 participantes, profesores en formación, estaban habituados a trabajar con las vistas algebraica, gráfica y hoja de cálculo, y poca experiencia en el uso de la Vista Gráfica $3 \mathrm{D}$. 


\section{Diseño de la Tarea y análisis a priori}

Para analizar el trabajo de los profesores en formación se diseñó una tarea donde se pregunta por calcular y visualizar (trazar) la curva en el espacio correspondiente al vector $\boldsymbol{r}(t)=$ $t \boldsymbol{i}+t^{2} \boldsymbol{j}+t^{3} \boldsymbol{k}$. Para ello, se propone que calculen para valores de $\mathrm{t}$, vectores particulares de la forma $\boldsymbol{r}\left(t_{0}\right)=t_{0} \boldsymbol{i}+t_{0}{ }^{2} \boldsymbol{j}+t_{0}{ }^{3} \boldsymbol{k}$, y también expresados como $\left(t_{0}, t_{0}{ }^{2}, t_{0}{ }^{3}\right)$. Antes de responder a la tarea, los profesores tuvieron oportunidad de revisar el tema correspondiente a funciones vectoriales propuesto en Stewart (2012). Las principales secciones de la tarea son las siguientes:

i. Calcular vectores particulares de la función vectorial propuesta y expresarlo de las formas $\boldsymbol{r}\left(t_{0}\right)=t_{0} \boldsymbol{i}+t_{0}{ }^{2} \boldsymbol{j}+t_{0}{ }^{3} \boldsymbol{k}, \mathrm{y}\left(t_{0}, t_{0}{ }^{2}, t_{0}{ }^{3}\right)$. Así como en la forma general $\left(t, t^{2}, t^{3}\right)$. Es importante mencionar que en esta primera sección no se explicita usar GeoGebra; aunque no se restringe.

ii. Graficar, en GeoGebra los vectores calculados.

iii. Analizar en términos de la curva asociada a la función vectorial, los puntos (vectores) de la forma $\left(t_{0}, t_{0}{ }^{2}, t_{0}{ }^{3}\right)$.

iv. Utilizar GeoGebra para calcular tantos puntos (vectores) de la forma $\left(t_{0}, t_{0}{ }^{2}, t_{0}{ }^{3}\right)$ como fuera posible. Previamente se solicita exponer qué herramientas de GeoGebra son útiles para esta tarea.

v. Finalmente, se pregunta si al utilizar GeoGebra es necesario hacer algún ajuste técnico a la herramienta utilizada.

En términos del marco teórico, las secciones de que se compone la tarea serán viables para observar cómo las características de la herramienta GeoGebra influyen en las formas de pensar del usuario sobre el trazo de curvas; de la misma manera, se pretende observar cómo las concepciones y conocimientos matemáticos dirigen la forma de uso de GeoGebra. En la primera sección, si bien la función vectorial es simple, interesa observar si de manera espontánea utilizan el software para calcular los vectores particulares solicitados. En la segunda sección, interesa observar qué herramientas de GeoGebra utilizan para visualizar los vectores. En la tercera y cuarta, el propósito es observar cómo utilizan GeoGebra para visualizar tantos vectores como se pueda en la forma $\boldsymbol{r}\left(t_{0}\right)=t_{0} \boldsymbol{i}+t_{0}{ }^{2} \boldsymbol{j}+t_{0}{ }^{3} \boldsymbol{k}$ y por lo tanto, la curva en el espacio. Por último, la sección cinco, se propone para observar de manera explícita si, en términos del marco teórico, 
el conocimiento de los profesores en formación sobre los vectores, modifica las formas de uso de las herramientas de GeoGebra.

Con base en el diseño de la tarea, a continuación se presentan formas posibles de uso de herramientas GeoGebra para visualizar la curva en el espacio de la función vectorial $\boldsymbol{r}(t)=$ $t \boldsymbol{i}+t^{2} \boldsymbol{j}+t^{3} \boldsymbol{k}$. Una primera forma de uso de GeoGebra es utilizar la herramienta deslizador para visualizar diferentes puntos de la forma $\left(t_{0}, t_{0}{ }^{2}, t_{0}{ }^{3}\right)$ asociando el valor del deslizador y a cada una de las componentes del vector; a partir de ello, es posible visualizar la curva a través de la herramienta trazo (Figura 1). Esta forma de uso, la identificamos como deslizador+rastro.

Figura 1: Visualización de la curva en el espacio mediante deslizador+ rastro

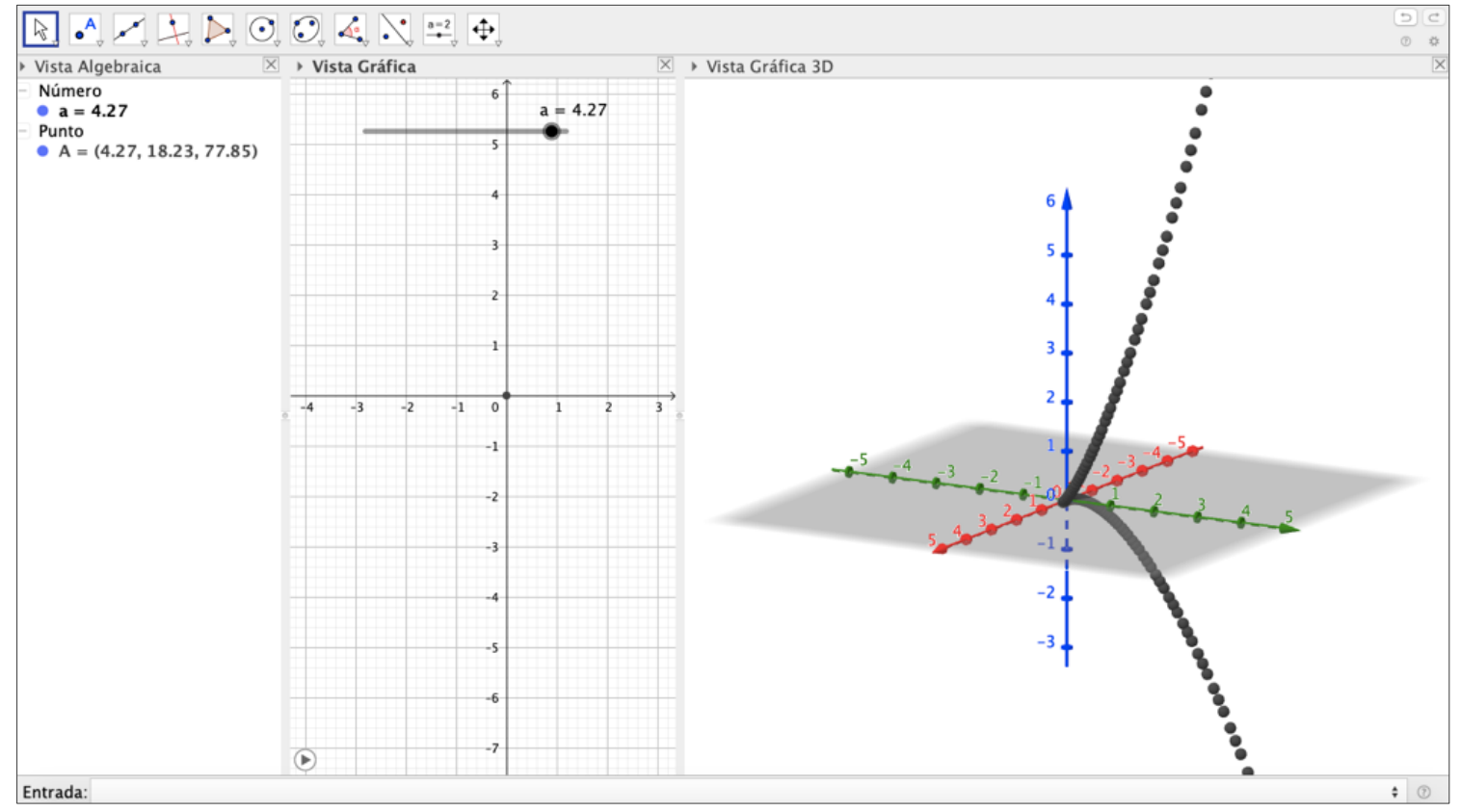

Fuente: Elaboración propia (2020)

Como explícitamente se solicita en la tarea, calcular vectores particulares y graficarlos, se espera que los futuros profesores visualicen también tales vectores mediante el uso directo de la herramienta vector, para lo cual se requiere el punto inicial y punto final; o bien escribir en la línea de entrada el comando Vector(Punto inicial, Punto final).

Una segunda forma para visualizar en GeoGebra la curva en el espacio (ventana 3D) consiste en una forma similar a la anterior: utilizar la herramienta deslizador para visualizar las componentes del vector dado, asociando el valor del deslizador a las componentes del vector de acuerdo con la función dada, sin embargo, en lugar de utilizar el rastro del punto final del vector, 
es posible utilizar la herramienta lugar geométrico (Figura 2). A esta forma de uso de GeoGebra, en este trabajo se identifica como deslizador+lugar geométrico.

Figura 2: Visualización de la curva en el espacio mediante deslizador+lugar geométrico

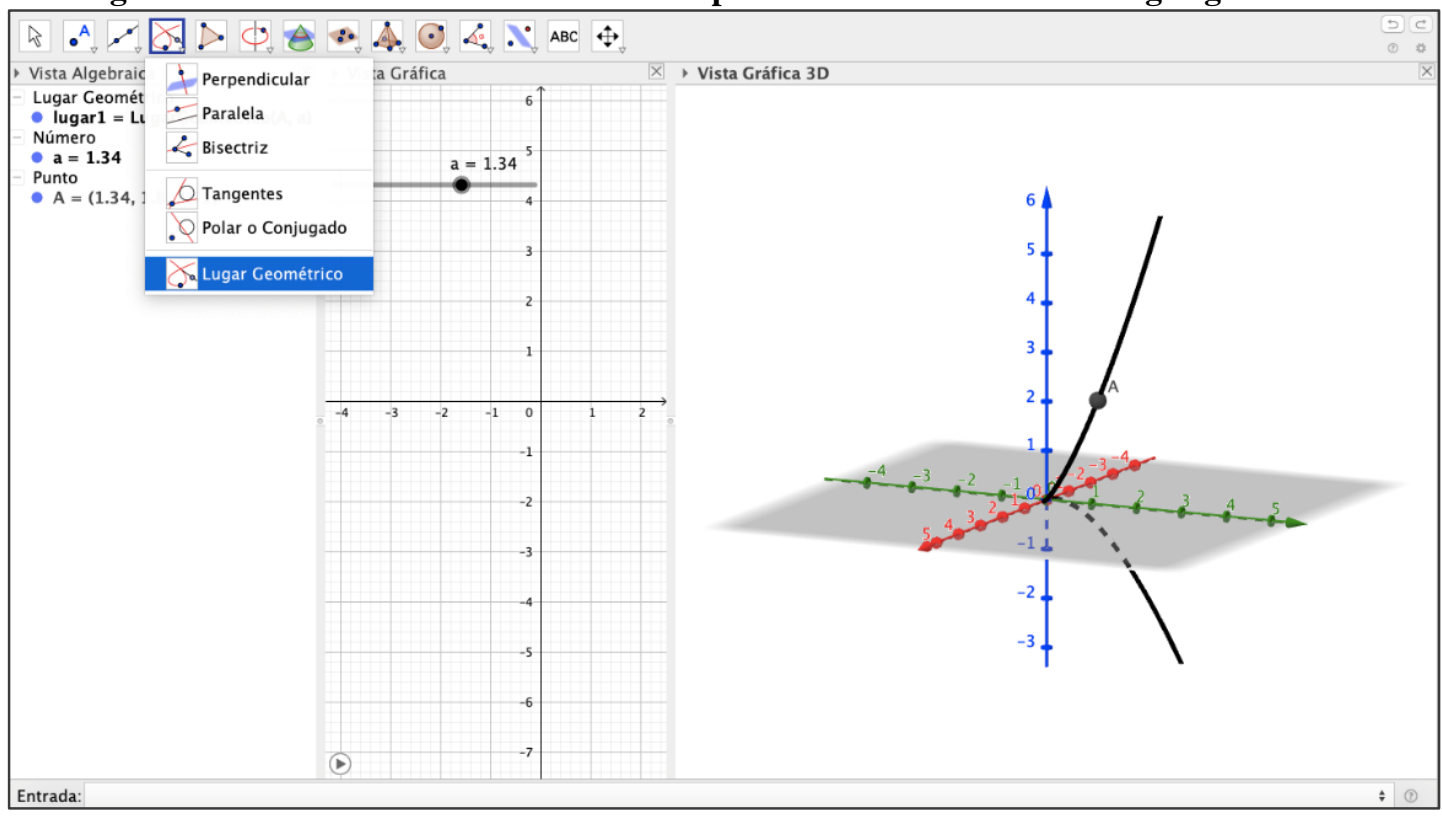

Fuente: Elaboración propia (2020)

La forma de visualizar la curva de la función vectorial indicada en la Figura 1, es decir, utilizando el rastro se trata de una visualización que no es fija, aunque indica, en cierta forma, la propiedad de continuidad de la función. Sin embargo, la característica que se explota en esta forma de uso es el dinamismo en la construcción de la curva (el rastro). En cambio, en la Figura 2, al utilizar la herramienta Lugar Geométrico (junto con la herramienta deslizador), el dinamismo asociado a la construcción de la curva se pierde, ya que si bien el punto inicial $A$, puede ser desplazado a lo largo de la curva mediante el deslizador, esta forma de construcción de la curva (mediante el lugar geométrico) no permite visualizar el recorrido del punto final del vector, ya que el comando Lugar Geométrico muestra en la ventana 3D la curva de forma instantánea. Sin embargo, la propiedad de continuidad de la función (visualizada en la curva) queda mejor representada, estas formas de trabajo son importantes a considerar de acuerdo con Gómez-Chacón, Botana, Escribano y Abánades (2016). Por otro lado, la idea de continuidad y dominio de la función puede asociarse al incremento y valores mínimo y máximo del deslizador, respectivamente. 
Una tercera forma de trabajo consiste en directamente escribir las ecuaciones paramétricas de la curva, es decir, expresión de la forma $\left(t, t^{2}, t^{3}\right)$ en la línea de entrada de GeoGebra. Esta forma de uso de GeoGebra produce lo que se muestra en la Figura 3. Esta tercera forma no considera ningún carácter dinámico en la construcción, sin embargo, la continuidad de la función vectorial es reconocida en esta construcción. Esta forma de trabajo es poco probable que se manifieste en los participantes, ya que este comando no ha sido utilizado por ninguno de los participantes, sin embargo, en su exploración de las formas de visualizar la curva, este puede emerger, además, la revisión del tema en el libro de texto explícita la ecuaciones paramétricas como $x=f(t), y=g(t), z=h(t)$, por lo que pueden asociar la expresión $\left(t, t^{2}, t^{3}\right)$ con estas ecuaciones.

Figura 3: Visualización de la curva mediante la escritura directa de las ecuaciones paramétricas en la línea de entrada

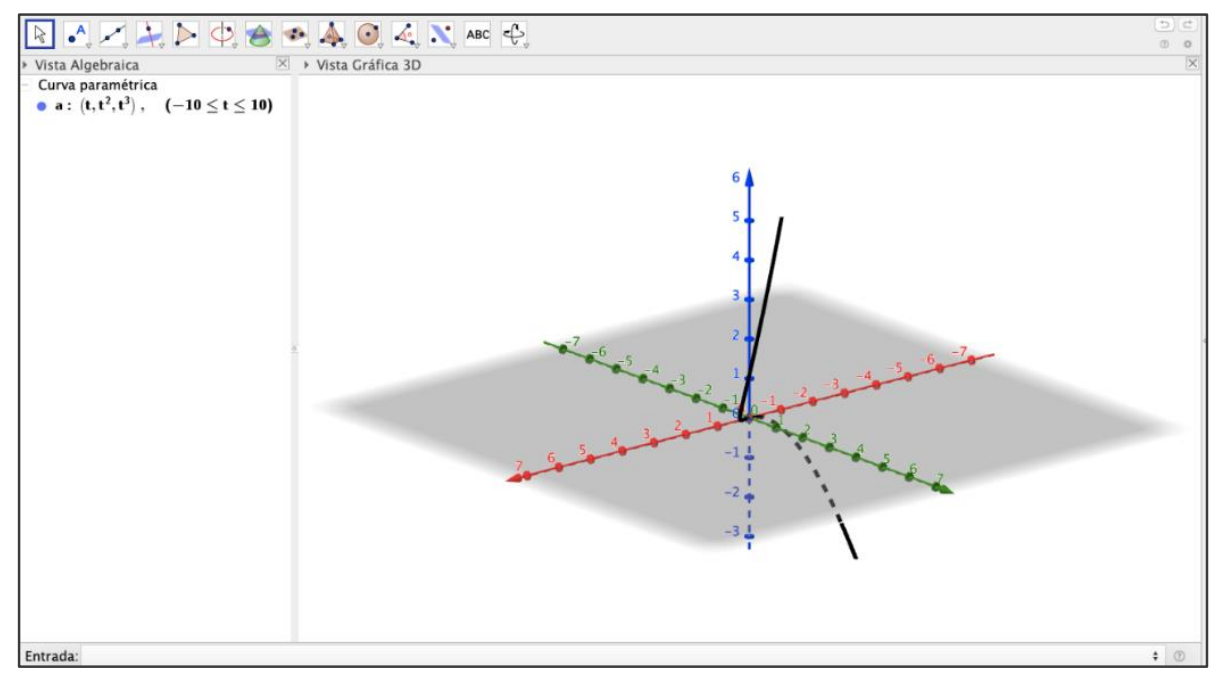

Fuente: Elaboración propia (2020)

Por último, otra forma de uso es representar los vectores en la hoja de cálculo cuyas componentes son de la forma $\left(t, t^{2}, t^{3}\right)$ y posteriormente visualizarlas en la vista gráfica (y algebraica). En esta forma de uso es difícil visualizar el carácter continuo de la función vectorial. La herramienta principal utilizada aquí es la hoja de cálculo+crear lista de puntos. Este tipo de trabajo se indica en la Figura 4. Por el carácter discreto evidente en la hoja de cálculo, la visualización de la curva es pobre en términos de la continuidad de la función vectorial. Sin embargo, esta forma de trabajo puede presentarse por la familiaridad que los participantes tienen con el trabajo en la hoja de cálculo de GeoGebra. La continuidad y dominio de la función en 
esta forma de trabajo se puede observar en el tipo de valores utilizados en la hoja de cálculo para el parámetro $t$.

Figura 4: Uso de la hoja de cálculo+crear lista de puntos para visualizar la curva de la función vectorial

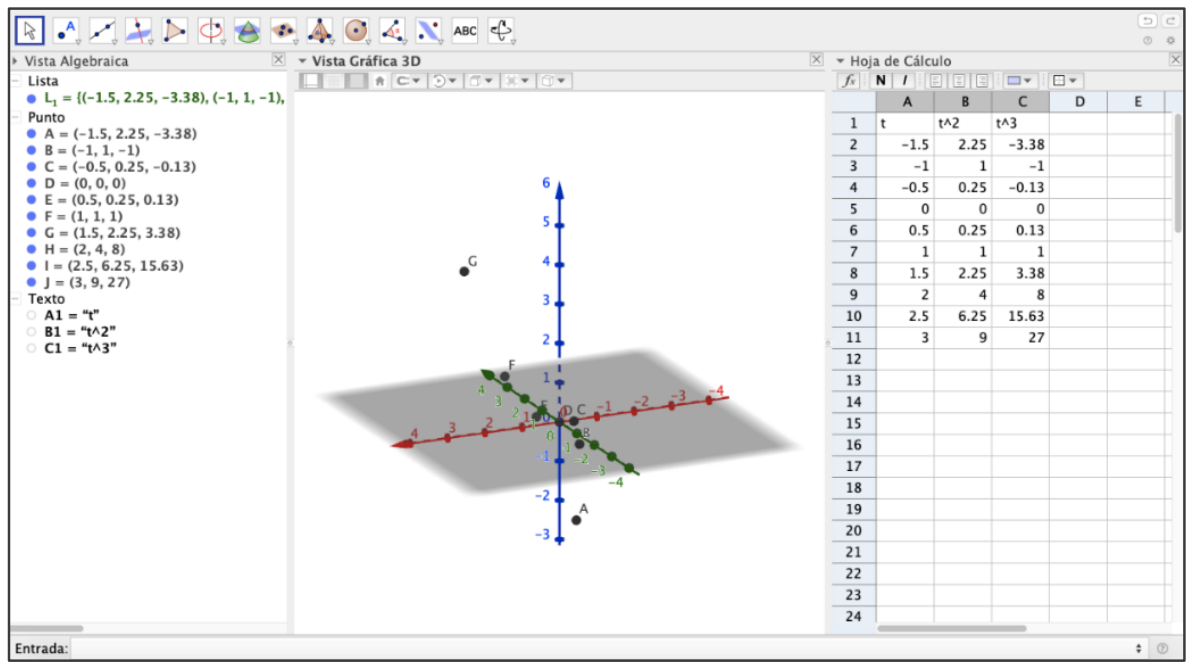

Fuente: Elaboración propia (2020)

Dependiendo de la forma de uso de la hoja de cálculo, el carácter discreto puede ampliarse o reducirse, sin embargo, esta forma tiene desventajas obvias (para representar la continuidad de la función) respecto a las tres formas de trabajo previas. Como ya fue mencionado, las construcciones de los participantes explicitarán la visualización de los vectores, porque así es solicitado en la tarea propuesta.

Respecto al uso del deslizador, una característica importante de éste, consiste en la viabilidad de modificar sus parámetros, por ejemplo, el incremento y valores mínimo y máximo que puede tomar, el primero está asociado a la idea de continuidad, el segundo al dominio de la función. Este tipo de características y potencialidades de la herramienta tecnológica se pretenden observar en el trabajo de los participantes, de la misma manera el objetivo es dar cuenta de cómo las concepciones y conocimientos matemáticos del profesor en formación dan forma al uso de las herramientas y comandos de GeoGebra.

\section{Análisis de datos y discusión de resultados}

A continuación, se presenta el análisis de datos y discusión de resultados, llevado a cabo con base en la hojas de trabajo de los profesores en formación, los archivos electrónicos generados (Archivos GeoGebra), la videograbación de la sesión en línea (realizada bajo el 
consentimiento de los participantes) y notas de campo del investigador. De los 16 participantes, 12 de ellos (Profesores en formación, PF1, PF2, PF3, etc., en adelante) completaron de manera individual la tarea, los 16 participantes formaron parte de la entrevista grupal. En este trabajo, el análisis se enfoca principalmente en las formas de uso de las herramientas GeoGebra observadas, respecto al procedimiento empleado así como posibles ajustes técnicos a las herramientas del software. Estas características en la actividad matemática ligada al uso de la herramienta, representan el tipo de trabajo de los participantes.

El análisis se divide en tres momentos, el primero de ellos sobre las formas de visualizar en la ventana 3D los vectores particulares solicitados en la tarea. El segundo refiere a la forma de visualizar las curvas en la ventana 3D. El tercero, a posibles ajustes de las herramientas.

\section{Visualización de vectores en la ventana $3 D$}

Los participantes que completaron la tarea siguen el mismo tipo de procedimiento para visualizar los vectores en la vista gráfica 3D. Once participantes escriben directamente en la línea de entrada cada vector particular de la forma $\left(t_{0}, t_{0}{ }^{2}, t_{0}{ }^{3}\right)$, el cual se representa en GeoGebra como un punto de coordenadas $(x, y, z)$, y es interpretado como el punto final del vector con origen en $(0,0,0)$. La siguiente Figura 5 muestra la construcción llevada a cabo por PF6 en GeoGebra (algunos participantes utilizaron el software GeoGebra en línea, otro el instalado en sus dispositivos); así como la explicación que ofrece del proceso seguido, ésta es del mismo tipo que indican los demás participantes. 
Figura 5: Construcción desarrollada por PF6 para visualizar vectores particulares de la función vectorial dada y explicación del proceso seguido

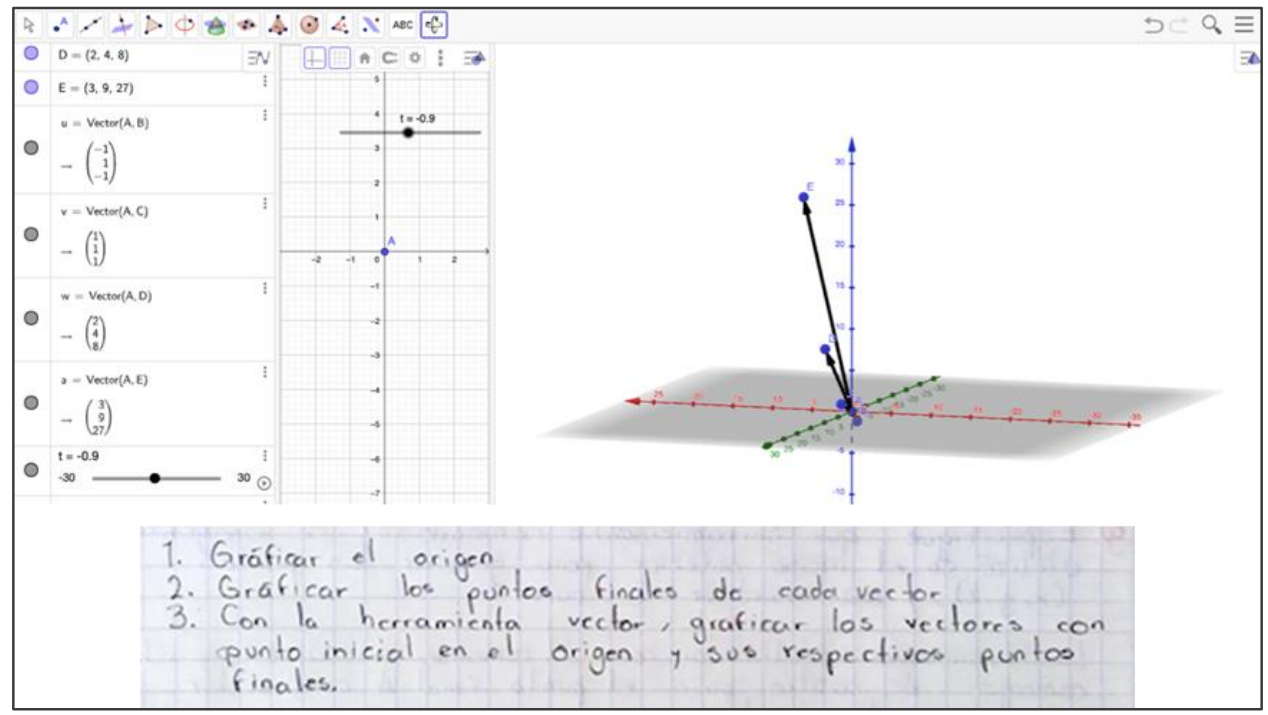

Fuente: Datos de la investigación (2020)

Por su parte, PF3 hace un procedimiento similar, pero en lugar de utilizar el punto inicial y final del vector, escribe en la línea de entrada el comando: vector (Punto). Este le permite visualizar el vector cuyo punto final es el punto referido en el comando, y como punto inicial en automático el software lo toma como el origen del sistema tridimensional.

\section{Visualización de curvas en la ventana $3 D$}

Las formas de trabajo identificadas en el trabajo de los participantes para visualizar las curvas en el espacio asociadas a la función vectorial, son las siguientes:

\section{$\underline{\text { Uso de deslizador+ rastro }}$}

Una de las formas de trabajo identificadas y con mayor frecuencia utilizada por los participantes (11 de ellos) refiere a utilizar las herramientas deslizador y rastro, a partir de asociar el movimiento del punto final del vector a un deslizador, el cual a su vez está vinculado a las componentes del vector. Sin embargo, aunque las explicaciones, en algunos casos, como PF3, no hacen alusión al uso del rastro, sólo del deslizador, en sus construcciones sí se observa que utilizan la herramienta rastro. La siguiente Figura 6, muestra la explicación escrita por PF6 (izquierda) y PF3 (derecha) para trazar la curva solicitada. 
Figura 6: Explicación sobre el trazo de la curva en el espacio tridimensional

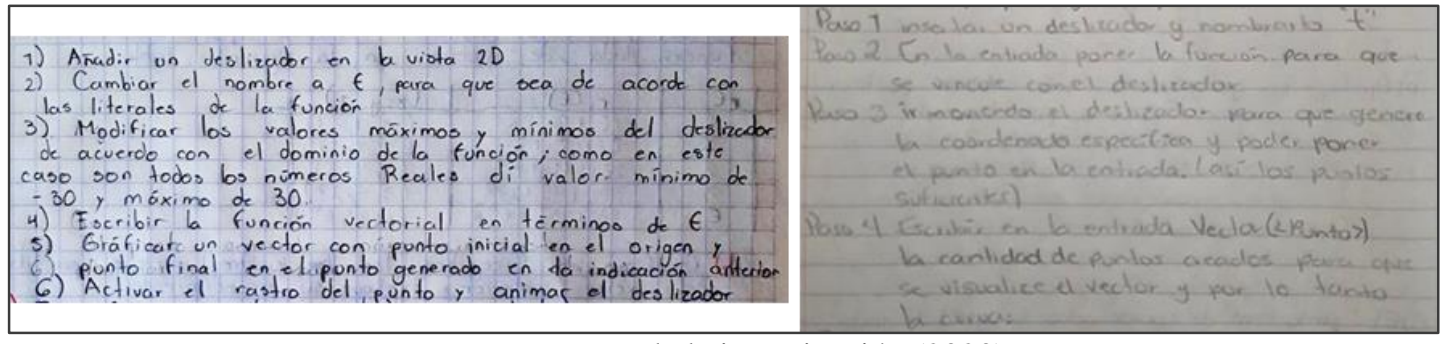

Fuente: Datos de la investigación (2020)

En la explicación de PF6 claramente se observa cómo las restricciones y posibilidades del software GeoGebra (deslizador y rastro particularmente) permiten al usuario utilizar esta estrategia para trazar la curva de la función vectorial. El proceso descrito por PF6 y PF3, permite una construcción de la curva como se muestra en la Figura 7. En esta se observa cómo las herramientas principales utilizadas son el deslizador, el rastro (del punto F) y el trazo del vector con punto final $\mathrm{F}$ y punto inicial en el origen (punto $\mathrm{A}$ ).

Figura 7: Forma de uso de GeoGebra por PF1 para visualizar la curva de la función vectorial

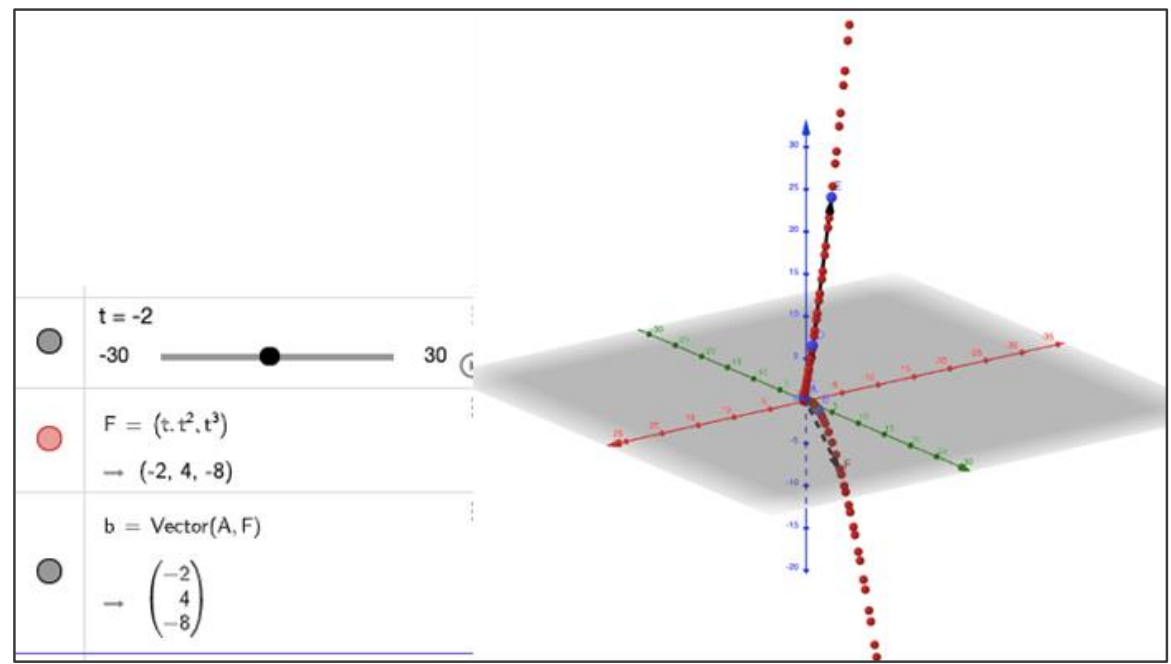

Fuente: Datos de la investigación (2020)

Respecto a la forma de los puntos que aparecen en la ventana 3D, al graficar los vectores particulares, y en este caso al visualizarlos mediante el rastro de un punto (como el punto $\mathrm{F}$ en la figura previa), los participantes explican la lógica de lo observado en GeoGebra, por ejemplo, como se observa en el siguiente extracto de la entrevista.

Entrevistador: ¿Alguien más? Interrumpí a alguien más, creo.

PF2: Yo, puse una idea similar a la de PF5, pero, puse que los puntos se generan a partir de la regla de correspondencia dada, o sea la función que los genera, la curva. Por lo tanto cada 
uno de esos puntos [Refiriéndose a los puntos visualizados en GeoGebra] van a irlos formando [se refiere a formar la curva].

Esta forma de trazar las curvas, da muestra de cómo la herramienta, sus potencialidades y restricciones, influye en la forma de pensar de los participantes. Por otro lado, los conocimientos del futuro profesor sobre el dominio de la función le conducen a utilizar GeoGebra en formas particulares, esto se discute más adelante. Otro elemento que se observa es cómo nombran al deslizador, le asignan la misma literal que el utilizado para el parámetro de la función vectorial. Sin embargo, también manifiestan que ello no es necesario, es decir, utilizar la literal que en automático asigna GeoGebra al trazar un deslizador para asociarla a las componentes del vector. Esto último fue manifestado por PF12.

\section{$\underline{\text { Uso de Hoja de Cálculo+Crear lista de puntos }}$}

Otra forma de trabajo observada en los estudiantes, específicamente en PF9, consiste en calcular las componentes del vector en una hoja de cálculo, y después hacer la conversión hacia el registro gráfico. Durante la entrevista grupal, PF9 menciona que la idea de su procedimiento es mantener siempre visible las componentes de los diferentes vectores que forman la curva, aspecto que no puede visualizarse cuando se utiliza un deslizador. Sin embargo, como veremos más adelante, este argumento es refutado por PF3. La siguiente Figura 8, muestra el procedimiento seguido por PF9 para visualizar la curva.

Figura 8: Procedimiento empleado por PF9 para visualizar la curva en la ventana 3D

Utilizando la hoja de cálculo ponemos 4 columnas donde una serán de las
variables y las otras de las imágenes, ingresamos en la primer y segunda fila
dos variables consecutivas ( 1 y 2 ) y en la primer fila de la otra columna
ingresamos la función x(t) y así las otras dos con y e z, se pondrá el nombre
de la casilla de la variable de cada fila, así se puede hacer el procedimiento
tanto como quieras. Puedes copiar las coordenadas arrojadas y graficarlo, y
para visualizarlos usar la herramienta desplazar vista gráfica.

Fuente: Datos de la investigación (2020)

Como se muestra en la Figura 8, el procedimiento planteado por PF9 se basa en considerar no solo los puntos finales de los vectores en la vista gráfica, sino tener una representación numérica de los mismos en la hoja de cálculo. Este argumento de PF9 es tomado en cuenta por PF3, para proponer, durante la entrevista grupal, una modificación a su forma de usar la herramienta de manera inicial, lo cual en este trabajo identificamos como una tercera forma de uso de GeoGebra; planteada en la siguiente sección. Respecto al uso de la hoja de 
cálculo, comparado con el uso del deslizador, para generar puntos de la curva, los participantes indican como válidos, matemáticamente, ambos procedimientos.

Entrevistador: ¿Qué sería más válido, matemáticamente, o los dos [Refiriéndose a utilizar el deslizador+rastro, o la hoja de cálculo] son lo mismo, no importa [cuál utilizar]?

PF14: Yo creo que se pueden utilizar las dos, como usted dice. El deslizador puede ser para ver los puntos de manera más general, y la tabulación para ver puntos específicos o particulares que queremos tratar sobre el tema.

Entrevistador: ¿Alguien más?

PF1: Yo considero que el deslizador es para mostrar lo que se hizo en la hoja de cálculo. [...] Entrevistador: ¿Por qué optaron por deslizador [más rastro]? y, ¿PF9, por qué optaste por no usar deslizador, sino una hoja de cálculo?

PF14: [...] Aunque también hemos usado hojas de cálculo con usted, por que es lo que teníamos más reciente [Refiriéndose al uso de la herramienta deslizador en sesiones previas al tema de funciones vectoriales]

PF2: Porque es lo más rápido, para poder visualizar la curva.

[...]

Entrevistador: ¿PF9, usted por qué no uso el deslizador?

PF9: Yo, aparte de poner la representación gráfica, que es poner ahí la función y los puntos, también lo quería hacer con la representación, como verlo numéricamente, o sea, utilizar entonces la tabla [...] No nada más poner el punto y que se mueva, sino que se vean también los valores en una tabla.

Como se observa en el extracto de la entrevista, los participantes toman como validos los dos procedimientos. Por un lado, al deslizador le asocian cierta generalidad, es decir, con tal herramienta se puede generar una familia de vectores y cada uno de ellos genera un punto que pertenece a la curva en el espacio. Por ejemplo, cuando PF2 menciona "Porque es lo más rápido para visualizar la curva" refiere a que mediante el deslizador y el rastro, se puede visualizar gran cantidad de puntos de la curva, es decir, lo general está asociado a visualizar "muchos" puntos. Por otro lado, el uso de la hoja de cálculo lo asocian a casos particulares de vectores. Más aún, los casos particulares pueden ser verificados, gráficamente mediante la estrategia deslizador+rastro, como lo menciona PF1. Sin embargo, para PF9, es importante mostrar a la vez la representación gráfica y numérica de los vectores (y por lo tanto, de la curva).

Como puede observarse, las ideas matemáticas de los participantes les conducen a utilizar las herramientas de GeoGebra en dos formas, inicialmente: primero, mostrar un mayor número de puntos de la curva mediante el deslizador, ello es posible porque el potencial de GeoGebra para esta herramienta así lo permite, por ejemplo, debido al incremento de los valores que toma el deslizador de manera automática. Segundo, visualizar los vectores (y la curva de la función) de manera simultánea en dos representaciones, la gráfica y la numérica. Ello es posible 
mediante la hoja de cálculo y la vista gráfica 3D. PF9, en sus palabras, lo resumen de la siguiente manera: "No es solamente un modelo gráfico, sino también un modelo numérico". Además de visualizar en una tabla las componentes de las imágenes de la función vectorial, para PF9 es una forma viable de representar gráficamente varios vectores, y "no sólo uno que se va moviendo”. Aquí se puede observar como PF9 interpreta el uso del deslizador con la existencia de un solo vector, no con una familia de vectores. Sin embargo, esta idea es refutada por PF3, como a continuación se muestra.

PF9:En el deslizador, sólo te da un vector, que es el que te da el deslizador. Yo puedo tener 10 vectores, todos están apuntando a la misma función, a la misma curva en el espacio, mientras que en el deslizador, solamente vamos a tener un vector que esta apuntando y se está moviendo sobre esa curva, por eso, yo quise hacerlo con una tabla, para que también se vieran distintos vectores, no solamente uno.

Entrevistador: $O k$, de acuerdo

PF3: También utilizando el deslizador se podrían observar varios vectores

Entrevistador: ¿Cómo, PF3?

PF3: Sólo activamos en rastro del vector, se va ir visualizando diferentes vectores, cada uno de los que va haciendo [se refiere a los que se generan para cada valor del deslizador].

Lo comentado por PF3 en el diálogo anterior, se considera en este trabajo como procedimiento adicional, a partir del uso de GeoGebra. Esto se indica en la siguiente sección.

\section{$\underline{\text { Uso de deslizador + rastro (adecuación) }}$}

En esta forma de trabajo queremos enfatizar una adecuación que propone PF3, respecto al uso del rastro para visualizar la familia de vectores asociados, es decir, activar el rastro también para los vectores generados por el deslizador. La Figura 9, muestra la construcción en GeoGebra propuesta por PF3, en la cual se visualizan puntos de la curva a través de un deslizador y cada vector particular asociado, de acuerdo con la función dada. 
Figura 9: Construcción original generado por PF2 (izquierda). Construcción modificada donde se muestra el rastro de los vectores

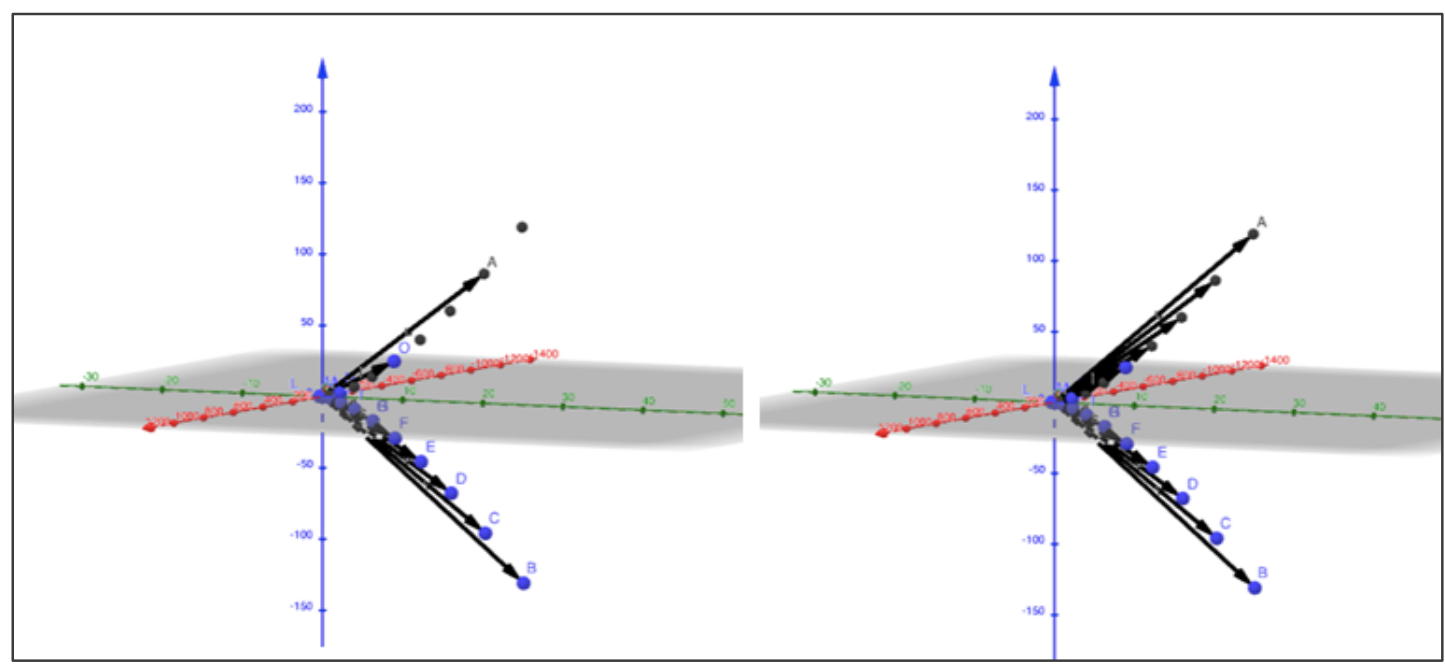

Fuente: Datos de la investigación (izquierda). Elaboración propia (derecha) (2020)

Los vectores trazados por PF2 (Figura 9 izquierda) corresponden a vectores particulares solicitados durante fase dos de la tarea; si bien en el archivo GeoGebra entregado por PF2 no se observa que haya activado el rastro como lo mencionó en la entrevista grupal, en la misma Figura 9 (derecha) se muestra la visualización de los vectores, mediante el rastro, a partir del mismo archivo de PF3, para indicar la sugerencia que hace. Los procedimientos descritos, hasta el momento, para visualizar los vectores particulares y la curva en el espacio, son los observados en los datos analizados referido al trabajo de los profesores en formación que completaron la tarea y durante la entrevista.

\section{Ajustes en el uso de herramientas de GeoGebra}

Las concepciones de los participantes, sus conocimientos matemáticos influyen en la forma de usar las herramientas de GeoGebra. Por ejemplo, cuando utilizan el deslizador, algunos participantes indican la necesidad de hacer ajustes en las propiedades de éste. Por ejemplo, cambiar el rango de valores máximo y mínimo del deslizador, ya que en palabras de PF6 "su dominio [el dominio de la función] son todos los [números] reales". Lo planteado por los participantes en cuanto a los ajustes necesarios, se observa en el siguiente extracto de la entrevista.

Entrevistador: ¿Hicieron algún ajuste, o usaron las herramientas de GeoGebra tal cual vienen de forma automática? 
PF6: Yo agregué más valores al deslizador [se refiere a que modificó el valor mínimo y máximo que puede tomar el deslizador], porque su dominio son todos los [números] reales.

Este tipo de cambios en los ajustes del deslizador, fue manifestado por tres participantes: PF4, PF6 y PF7. La Figura 10, muestra la explicación escrita por PF7, al respecto. Otro tipo de ajustes, mencionados por los participantes son respecto al incremento (es el caso de PF4) de los valores que puede tomar el deslizador, o bien ajustes en la ventana 3D, particularmente en los ejes coordenados (ver Figura 11).

Figura 10: Explicación de PF7 acerca de cambiar ajustes del deslizador

$$
\text { Modificai los valores Max y Min }
$$

$\frac{\text { del deslizador. }}{\text { Fuente: Datos de la investigación (2020) }}$

Figura 11: Explicación de PF5 sobre ajustes realizados en GeoGebra

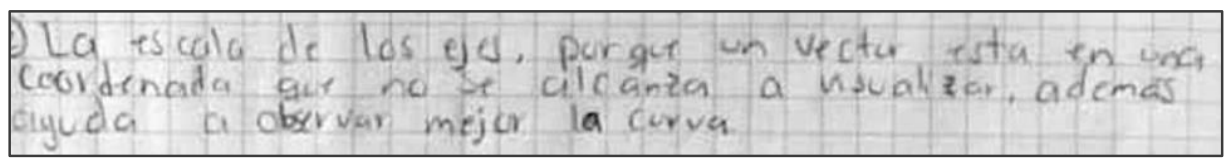

Fuente: Datos de la investigación (2020)

El tipo de ajuste mencionado por PF5, ha sido considerado importante cuando se utilizan medios tecnológicos para graficar. Por ejemplo, Trouche (2005), así como Trouche y Drijvers (2010) han indicado que el uso apropiado de la ventana gráfica es importante para una correcta interpretación y razonamiento sobre de las gráficas. Respecto a funciones vectoriales en $\mathrm{R}^{3}$, este tipo de ajuste resulta relevante, ya que la visualización de la curva puede hacerse desde distintas perspectivas. Más aun, de acuerdo con Rocha (2020), para los profesores, en nuestro caso, en formación, la identificación de ajustes necesarios en la ventana de graficación, es un aspecto relevante.

\section{Conclusiones y comentarios finales}

Desde el punto de vista de la génesis instrumental, en el trabajo desarrollado por los participantes es posible observar las componentes indicadas en este enfoque teórico. Respecto al proceso de instrumentación, la herramienta tecnológica ofrece al usuario ciertas posibilidades y restricciones con las que puede trabajar para generar vectores y la curva en el espacio de la función dada. Como fue planteado, los participantes optaron por el uso del deslizador y el rastro, particularmente para generar las curvas, además, uno de los participantes optó por el registro 
numérico y gráfico que permite GeoGebra para mostrar simultáneamente en dos registros, de manera estática, los vectores solicitados. Por otro lado, en cuanto al proceso de instrumentalización, las concepciones y conocimientos matemáticos permitieron guiar la forma de uso de las herramientas de GeoGebra. Por ejemplo, en el caso de PF6, su conocimiento sobre el dominio de las funciones y el aspecto de la continuidad de la función le conducen a usar de forma particular la herramienta deslizador a través de hacer ajustes de las propiedades que por default genera GeoGebra.

En este mismo sentido PF3, en el uso del deslizador, cambia algunas propiedades con que automáticamente se genera esta herramienta, en su caso es el incremento del deslizador, lo modifica a 0.5 ; con el cual, por lado se pierde la idea de continuidad, pero se visualizan de mejor manera los vectores generados. Por último en el caso del PF9, su idea de explícitamente visualizar de forma simultánea los vectores en su registro gráfico y numérico, le conducen a utilizar la Hoja de cálculo, la vista 3D y la vista algebraica.

Así, por un lado, las herramientas de GeoGebra influyen en la forma de pensar la curva que se observa en la ventana 3D, es decir, se trata del proceso de instrumentación. Con el uso del deslizador y rastro se observa un dinamismo en la construcción, propio de este tipo de software, lo que les conduce a interpretar como una representación gráfica adecuada en términos de la continuidad de la función, debido al número de vectores que se generan, y por lo tanto, puntos de la curva; asociados al incremento de los valores del deslizador. Además, esta forma de trabajo la asocian con una forma general, que se puede interpretar como ligado a la continuidad. En este sentido, estos resultados están en línea con lo planteado sobre el arrastre en la geometría dinámica, respecto a que desde un punto de vista epistemológico las restricciones y potencialidades de las herramientas tecnológicas y sus formas de uso pueden dar origen a nuevos significados (Trouche \& Drijvers, 2010). Mientras que el trabajo en dos registros, a través de la hoja de cálculo y la vista gráfica, lo asocian a una representación adecuada en términos de la familia de vectores que genera la curva, y que pueden visualizarse gráfica y numéricamente, al menos de manera distinta a cuando se utiliza el deslizador. En cuanto a los ajustes llevados a cabo, estos son dirigidos por su conocimiento matemático, es decir, se trata de la instrumentalización. Hacer ajustes debido al dominio de la función, su continuidad, o la escala de los ejes coordenados, es representativo de la instrumentalización. 
De esta forma, de acuerdo con la actividad matemática de los participantes en este tipo de ambiente tecnológico; es posible indicar que los profesores en formación desarrollaron las siguientes formas de uso de las herramientas de GeoGebra, que a la vez dan cuenta de los esquemas desarrollados con el uso de la tecnología: uso de deslizador y activación del rastro para generar curvas de funciones vectoriales (carácter discreto pero asociado a la continuidad), uso de la hoja de cálculo y la vista gráfica para visualizar de forma simultánea los vectores y la curva (carácter discreto) de la función vectorial, y por último, uso de deslizador y activación de rastro, para visualizar puntos de la curva del vector, así como de la familia de vectores mismos.

Tales formas de uso de las herramientas de GeoGebra son muestra del desarrollo, de forma simultánea, de la instrumentación e instrumentalización, es decir, del uso de la herramienta como instrumento y no solo como artefacto. Sin embargo, como se postula en el enfoque teórico, la evolución de un artefacto en instrumento, es un proceso dialéctico en desarrollo constante. Así, en una primera etapa, respecto al trazo de curvas de funciones vectoriales, el uso de la herramienta puede considerarse a nivel de instrumento, no así para las reflexiones ligadas a la continuidad. A este respecto, en tanto el trabajo en medios computacionales se está siempre en un mundo discreto, la continuación de la reflexión matemática y el uso de otras herramientas como el comando lugar geométrico es necesario en este tipo de población. Con lo mostrado en este trabajo, se da evidencia de la importancia de ofrecer oportunidades al futuro profesor para engancharse en reflexiones matemáticas en ambientes tecnológicos, para su propio aprendizaje.

Como se recordará, el enfoque teórico en que se basó el estudio fue el desarrollo de la génesis instrumental a nivel personal, por ello, no es posible en este trabajo discutir el desarrollo de génesis instrumental profesional, además de que la tarea diseñada para la recolección de datos no fue planteada para tal propósito. En este sentido, como investigaciones futuras, planteamos dos direcciones, la primera, respecto a la importancia del conocimiento sobre las características de las ventanas gráficas, o ventanas de visualización, ya que como lo indica Rocha (2020) se requiere de mayor literatura sobre el conocimiento para la enseñanza de las matemáticas con tecnología en este aspecto. Por otro, sustentados en planteamientos acerca de que en la formación de profesores, durante su aprendizaje matemático reflexionan también sobre la enseñanza de la matemática (Holm, 2019), es pertinente analizar si los profesores en formación, 
mientras reflexionan sobre su propio conocimiento matemático en ambientes tecnológicos, también lo hacen acerca la enseñanza mediada por tecnología.

\section{Referencias}

Bozkurt, G., Uygan, C. \& Turgut, M. (2018). Instrumental genesis of a preservice mathematics teacher: instrumented actions for perpendicular line construction in a dynamic geometry environment. En H.G. Weigand, A. Clarck-Wilson, et al. (Eds.), Proceedings of the 5th ERME Topic Conference MEDA. Copenague, Dinamarca: Universidad de Copenague. Disponible en: https://www.math.ku.dk/english/research/conferences/2018/meda/proceedings/papers/b ozkurt_p_43.pdf.

Clark-Wilson, A. Guilles, A., Cusi, A., Goos, M., Haspeskian, M. Robutti, O. \& Thomas, M. (2014). The challenges of teaching mathematics with digital technologies-the evolving role of the teacher. Ponencia presentada en el 38 Annual Meeting of the International Group for the Psychology of Mathematica Education. Disponible en: http://www.pme38.com/wp-content/uploads/2014/05/RF-Clark-Wilson-et-al.pdf. Acceso 20 de marzo de 2020.

Cory, B. L. \& Garofolo, J. (2011). Using Dynamic Sketches to enhance preservice secondary mathematics teachers' understanding of limits of sequences. Journal for Research in Mathematics Education, 42(1), 65-97. Disponible en https://www.researchgate.net/publication/259749831_Using_Dynamic_Sketches_to_E nhance_Preservice_Secondary_Mathematics_Teachers'_Understanding_of_Limits_of_ Sequences. Acceso 23 de marzo de 2020.

Drijvers, P. \& Trouche, L. (2008). From artifacts to instruments, a theoretical framework behind the orchestra metaphor. En G. W. Blume \& M. K. Heid (Eds.), Research on technology and the teaching and learning of mathematics: Vol. 2 cases, and perspectives (pp. 363391). Charlotte, NC: Information Age Publishing.

Gómez-Chacón, I. M., Botana, F., Escribano, J. \& Abánades, M. A. (2016). The concept of Locus. Genesis of personal and professional use with different tools. Bolema, 30(54), 67-94.

Haspekian, M. (2011). The co-construction of a mathematical and a didactical instrument. En M. Pytlak, T. Rowland, \& E. Swoboda (Eds.) Proceedings of of the 7th Congress of the European Society for Research in Mathematics Education (CERME7) (pp. 2298- 2307). Rzeszów: University of Rzeszów.

Hegedus, S., Laborde, C., Brady, C., Dalton, S., Siller, H-S., Tabach, M., Trgalova, J. \& Moreno-Armella, L. (2017). Uses of technology in upper secondary mathematics education. ICME-13 Topical Surveys. Suiza: Springer.

Holm, J. (2019). Supporting the development of future mathematics teachers: mathematics as healing. En S. Otten, A. G. Candela, Z. de Araujo, C. Haines, \& C. Munter (2019). Proceedings of the forty-first annual meeting of the North American Chapter of the 
International Group for the Psychology of Mathematics Education (pp. 1203-1207). St Louis, MO: University of Missouri.

Huang, R. \& Zbiek, R. M. (2017). Prospective Secondary Mathematics Teacher Education Preparation and Technology. En M. E. Strutchens, R. Huang, L. Losano, Potari D. et al. (Eds.), The Mathematics Education of Prospective Seconadry Teachers Around the World. ICME-13 Topical Surveys (pp. 17-24). Suiza: Springer.

Kieran, C. \& Drijvers, P. (2006). The co-emergence of machine techniques, paper-and- pencil techniques, and theoretical reflection: A study of CAS use in secondary school algebra. International Journal of Computers for Mathematical Learning, 11, 205-263.

Lobo da Costa, N. M., Galvao, M. E. \& Prado, M.E. (2017). Integration of Digital Technologies in Mathematics Teacher Education: The Reconstruction Process of Previous Trigonometrical Knowledge. En G. Aldon, F. Hitt, L. Bazzini \& U. Gellert (Eds.), Mathematics and Technology (pp. 523-550). Suiza: Springer.

Martínez-Hernández, C. \& Ulloa-Azpeitia, R. (2017). Dynamic Geometry Software and tracing tangents in the context of the mean value theorem: technique and theory production. International Journal for Technology in Mathematics Education, 24(2), 75-82.

Mishra, P., \& Koehler, M. J. (2006). Technological pedagogical content knowledge: A framework for integrating technology in teacher knowledge. Teachers College Record, 108(6), 1017-1054.

Rabardel, P. (2002). People and technology-a cognitive approach to contemporary instruments. Université Paris 8. Disponible en https://hal-univ- paris8.archivesouvertes.fr/file/index/docid/1020705/filename/people_and_technology.pdf. Acceso 10 de marzo de 2020.

Rocha, H. (2020). Graphical representations of functions using technology: a window to teacher knowledge. Teaching Mathematics and Its Aplictions. 39(2), 105-126.

Santos, J.A. \& Acuña, C.M. (2015). La tablet electronica, la genesis instrumental y los profesores de bachillerato. En R. Flores (Ed.), Acta Latinoamericana de Matemática Educativa Vol. (28). México: Comité Latinoamericano de Matemática Educativa AC.

Stewart, J. (2012). Cálculo de varias variables. Trascendentes tempranas. México: Cengage Learning.

Tabach, M. \& Trgalová, J. (2018). ICT standards for teachers: Toward a frame defining mathematics teachers' digital knowledge. En H-G. Weigand, et al. (Eds.), Proceedings of the 5th ERME Topic Conference. Mathematics Education in the Digital Age (pp. 273280). Dinamarca: Universidad de Copenhagen.

Trouche, L. (2005). Instrumental genesis, individual and social aspects. In D. Guin, K. Ruthven, \& L. Trouche (Eds.), The didactical challenge of symbolic calculators: Turning a computational device into a mathematical instrument (pp. 197-230). New York: Springer.

Trouche, L. \& Drijvers, P. (2010). Handheld technology for mathematics education: flashback into the future. ZDM, 42, 667-681.

Vasilachis, I. (2006). Estrategias de investigación cualitativa. Barcelona: Gedisa. 
Autores

Cesar Martínez Hernández

Profesor Investigador de Tiempo Completo de la Facultad de Ciencias de la Educación de la Universidad de Colima. Doctor en Ciencias en la Especialidad de Matemática Educativa por el Centro de Investigación y de Estudios Avanzados del IPN (Cinvestav-IPN). E-mail: cmartinez7@ucol.mx

Rodolfo Rangel Alcántar Profesor Investigador de Tiempo Completo de la Facultad de Pedagogía de la Universidad de Colima. Doctor en educación e innovación educativa, por la Universidad Virtual de Estudios Superiores, UNIVES. E-mail: rodolfo1@ucol.mx

José Marcos López Mojica Profesor de la Facultad de Matemáticas de la Universidad Autónoma de Guerrero. Doctor en Ciencias especialidad Matemática Educativa por el Centro de Investigación y de Estudios Avanzados del IPN (Cinvestav-IPN). E-mail: mojicajm@gmail.com

Norma Angélica Barón Ramírez Profesora Investigadora de Tiempo Completo de la Licenciatura en Pedagogía de la Universidad de Colima. Maestra en Ciencias, Área: Tecnología y Educación por la Universidad de Colima. E-mail: norma@ucol.mx 